\title{
Effect of dengue fever on the total leucocyte count and neutrophil count in children in early febrile period
}

\author{
Khandelwal $\mathbf{R}^{1}$, Khandelwal $\mathbf{L M}^{2}$ \\ ${ }^{1}$ Dr. Rohit Khandelwal, Assistant Professor, Department of Paediatrics, Vydehi Institute of Medical Sciences and \\ Research Centre, Bangalore, ${ }^{2}$ Dr. Leeni Mehta Khandelwal, Consultant Physician, Department of Internal \\ Medicine, Apollo Clinic and Hospital, Bangalore, Karnataka, India.
}

Address for Correspondence: Dr. Rohit Khandelwal, E-mail: docr109@gmail.com

\begin{abstract}
Introduction: Dengue is an arboviral disease caused by flavivirus. In India, the incidence of dengue fever is on a rise every year. The course of illness is different for each patient. Therefore early measurement of laboratory parameters can guide to the management of the disease. Aim of the study: 1) To study the effect of dengue fever on the total leucocytecount (TLC) in children in early febrile period. 2) To determine the neutrophil count in early stage of dengue fever. Study design: Observational study. Methodology: Patients of 1-14 years age group, who presented with fever of recent duration were included in the study. About 80 patients with early manifestations (15 days) of fever were included. Dengue was suspected in 55 children who had two or more of the following symptoms- fever, pain abdomen, headache, myalgias, arthralgias, skin rash, nausea, vomiting, retroorbital pain, prostration. Out of these 55 children, 50 children who presented in early febrile period (1-5 days) and who were serologically positive for denguetook part in the study. Blood investigations were done for total leucocyte count (TLC) and neutrophil count.Results: About $26 \%$ of the patients had leucopenia $(<4000), 66 \%$ had leucocyte count in normal range $(4,000-11,000)$ and only $8 \%$ had leukocytosis $(>11,000)$. About $34(68 \%)$ patients had neutrophil count of less than $40 \%$ and 16 (32\%) patients had neutrophils between 40 to $80 \%$. Conclusion: In early febrile period of dengue fever, majority have normal white blood cell count. Any change in the values of total leucocyte count points towards the progression of the disease towards severity. There is neutropenia in most of patients in dengue fever. Presence of neutropenia merely doesn't determine the use of antibiotics.
\end{abstract}

Keywords: Dengue, Leucocyte, Neutrophils

\section{Introduction}

Dengue virus belongs to family Flaviviridae, having four serotypes that spreads by the bite of infected Aedesmosquitoes [1]. There are 4 distinct, but closely related, serotypes of the virus that cause dengue (DEN-1, DEN-2, DEN-3 and DEN-4). Recovery from infection by one provides lifelong immunity against that particular serotype. However, cross-immunity to the other serotypes after recovery is only partial and temporary. Subsequent infections by other serotypes increase the risk of developing severe dengue [2].

Based on neutralization assay data, four serotypes (DENV-1, DENV-2, DENV-3, and DENV-4) can be

Manuscript received: $4^{\text {th }}$ October 2017

Reviewed: $14^{\text {th }}$ October 2017

Author Corrected: $20^{\text {th }}$ October 2017

Accepted for Publication: $26^{\text {th }}$ October 2017 distinguished. Dengue virus (DENV) infection is a major cause of disease in tropical and subtropical areas, with an estimated 50 million infections occurring each year and more than 2.5 billion people being at risk of infection. Infection with any of the DENV serotypes may be asymptomatic in the majority of cases or may result in a wide spectrum of clinical symptoms, ranging from a mild flu-like syndrome (known as dengue fever [DF]) to the most severe forms of the disease which are characterized by coagulopathy, increased vascular fragility, and permeability (dengue hemorrhagic fever [DHF]).

The latter may progress to hypovolemic shock (dengue shock syndrome [DSS])[3]. Severe dengue (also known as Dengue Haemorrhagic Fever) was first recognized in the $1950 \mathrm{~s}$ during dengue 
epidemics in the Philippines and Thailand. Today, severe dengue affects most Asian and Latin American countries and has become a leading cause of hospitalization and death among children and adults in these regions [2].

As the early presentations of DF and DHF/DSS are similar and the course of infection is short, timely identification of persons that will develop severe manifestations can be challenging. There is a longstanding debate as to whether DHF/DSS represents a separate pathophysiological process or is merely the opposite end of a continuum of the same illness. DF follows an uncomfortable but relatively benign self-limited course. DHF may appear as a relatively benign infection at first but can quickly develop into life-threatening illness as fever abates. DHF can usually be distinguished from DF as it progresses through its three predictable pathophysiological phases:

- Febrile phase: Viremia-driven high fevers

- Critical/plasma leak phase: Sudden onset of varying degrees of plasma leak into the pleural and abdominal cavities

- Convalescence or reabsorption phase: Sudden arrest of plasma leak with concomitant reabsorption of extravasated plasma and fluids

For optimal management of the patient with dengue infection, it is important to understand these phases and to be able to distinguish DHF from DF. Early recognition of a patient's clinical phase is important in order to tailor clinical management, monitor effectiveness of the treatment, and to anticipate when changes in their management are needed. Anticipatory management and monitoring indicators are essential in effectively administering therapies as the patient enters the critical phase. New-onset leucopenia (WBC $<5,000$ cells $/ \mathrm{mm} 3$ ) indicate that the fever will likely dissipate within the next 24 hours and that the patient is entering into the critical phase [4].Thus the need of measuring total leucocyte count in early phase is necessary for detection of any severe cases.

Dengue virus infections are a serious cause of morbidity and mortality in most tropical and subtropical areas of the world; Southeast and South Asia,Central and South America, and the Caribbean. Dengue virus infection can be asymptomatic or causes two forms of illness, dengue fever (DF) and dengue hemorrhagic fever (DHF) [5]. The first reported case of dengue like illness in India was in Madras in 1780, the first virologically proved epidemic of DF in India occurred in Calcutta and Eastern Coast of India in 1963-1964 [1]. Dengue virus infection presents with a diverse clinical picture that ranges from asymptomatic illness to DF to the severe illness of dengue hemorrhagic fever/ dengue shock syndrome (DHF/DSS) [6].

Polymorphonuclear neutrophils (PMNs) are the most abundant leukocytes in the blood and constitute the first line of host defense against numerous infectious pathogens, including bacteria, fungi, and protozoa. Neutrophils are the first leukocytes to migrate to sites of inflammation and infection where they recognize and phagocytose invading microorganisms, in order to kill them via different cytotoxic mechanisms. This process involves molecular mechanisms that coordinate cell polarization, delivery of receptors, and activation of integrins at the leading edge of neutrophils migrating toward chemoattractant. Once at sites of infection, neutrophils actively phagocytose microorganisms or form neutrophil extracellular traps (NETs) to trap and kill pathogens. Association of the nicotinamide adenine dinucleotide phosphate (NADPH) reduced oxidase complex at the phagosomal membrane for the production of reactive oxygen species (ROS) and delivery of proteolytic enzymes into the phagosome initiate pathogen killing and removal [7] $\mathrm{T}$.

\section{Aim of study}

1) To study the effect of dengue fever on the total leucocytecount (TLC) in children in early febrile period. 2) To determine the neutrophil count in early stage of dengue fever.

\section{Materials and Methods}

The study was conducted in the Department of Pediatrics of Vydehi Institute of Medical Sciences and Research Center, Bangalore from January 2015 to June 2015. A detailed history and a thorough clinical examination were done in all the cases. Data was collected in a prewritten proforma. Inclusion criteria: 1) Patients of 1-14 years age group. 2) Fever of recent duration. 3) Serological positivity for dengue. Exclusion criteria: 1) Febrile illness other than dengue. 2) Complicated dengue. About 
80 patients with early manifestations (1-5 days) of fever were included. Dengue was suspected in 55 children, who had two or more of the following symptoms: fever, pain abdomen, headache, myalgias, arthralgias, skin rash, nausea, vomiting and retroorbital pain. Out of these 55 children, 50 children who presented in early febrile period (1-5 days) and who were serologically positive for dengue took part in the study. Blood investigations were done for total leucocyte count (TLC) and neutrophil count. Patients were treated conservatively and with intravenous fluids and paracetamol. Vitals were monitored continuously. Patients were hemodynamically stable throughout the study. There was no mortality observed.

\section{Statistical Methods}

It was an observational study. Descriptive and inferential statistical analysis has been carried out in the present study. Results on continuous measurements are presented on Mean \pm SD (Min-Max) and results on categorical measurements are presented in Number (\%). Significance is assessed at $5 \%$ level of significance.

Student $\mathrm{t}$ test (two tailed, independent) has been used to find the significance of study parameters on continuous scale between two groups (Inter group analysis) on metric parameters. The Statistical software namely SAS 9.2, SPSS 15.0, Stata 10.1, MedCalc 9.0.1, Systat 12.0 and R environment ver.2.11.1 were used for the analysis of the data and Microsoft word and Excel have been used to generate graphs, tables etc.

Significant figures

+ Suggestive significance (P value: $0.05<\mathrm{P}<0.10$ )

* Moderately significant (P value: $0.01<\mathrm{P} \leq 0.05$ )

** Strongly significant ( $\mathrm{P}$ value: $\mathrm{P} \leq 0.01$ )

\section{Results}

The study was an observational study and included 50 patients who were serologically positive for dengue fever of recent onset. Age group of 1 - 14 years was included.

The study comprised of 19 females (38\%) and 31 males $(62 \%)$.

Table-1: Gender distribution of patients studied.

\begin{tabular}{|c|c|c|}
\hline Gender & No. of patients & \% \\
\hline Female & 19 & 38.0 \\
\hline Male & 31 & 62.0 \\
\hline Total & $\mathbf{5 0}$ & $\mathbf{1 0 0 . 0}$ \\
\hline
\end{tabular}

Table-2: Positive findings of NS1 and IgM in patients studied.

\begin{tabular}{|c|c|c|}
\hline & $\begin{array}{c}\text { No. of patients } \\
(\mathbf{n = 5 0 )}\end{array}$ & \% \\
\hline NS1 & 42 & 84.0 \\
\hline IgM & 26 & 52.0 \\
\hline
\end{tabular}

In thisstudy, out of the total 50 patients with positive dengue test, $42(84 \%)$ patients were found to be NS1 positive and $26(52 \%)$ showed IgM positive.

Table-3: Blood counts of patients studied.

\begin{tabular}{|c|c|c|c|}
\hline & $\begin{array}{l}\text { No. of patients } \\
\qquad(\mathrm{n}=50)\end{array}$ & $\%$ & Mean \pm SD \\
\hline \multicolumn{4}{|c|}{ Total LeukocyteCount(per cubic millimeter) } \\
\hline - $<4000$ & 13 & 26.0 & \multirow{3}{*}{$6480.74 \pm 3342.41$} \\
\hline - 4000-11000 & 33 & 66.0 & \\
\hline - $>11000$ & 4 & 8.0 & \\
\hline
\end{tabular}


Table-4: Comparison of Clinical variables according to age in years.

\begin{tabular}{|c|c|c|c|c|}
\hline \multirow{2}{*}{ Variables } & \multicolumn{2}{|c|}{ Age in years } & \multirow{2}{*}{ Total } & \multirow{2}{*}{ P value } \\
\cline { 2 - 5 } & $\mathbf{1 - 6}$ yrs & $\mathbf{7 - 1 4}$ yrs & & $0.076+$ \\
\hline $\begin{array}{c}\text { Total Leucocyte } \\
\text { Count }\end{array}$ & $4805.00 \pm 2388.68$ & $6899.68 \pm 3437.95$ & $6480.74 \pm 3342.41$ & 0.45 \\
\hline
\end{tabular}

In the early febrile period, the white blood cells in both the age groups (1-6 years and 7-14years) was within normal limits with an insignificant 'p' value.

Table- 5: Differential counts of patients studied.

\begin{tabular}{|l|c|c|c|}
\hline & $\begin{array}{c}\text { No. of patients } \\
(\mathbf{n = 5 0 )}\end{array}$ & \% & \multirow{2}{*}{ Mean \pm SD } \\
\cline { 1 - 3 } Differential count- Neutrophils & & 68.0 & \multirow{2}{*}{$42.52 \pm 14.66$} \\
\cline { 1 - 3 }$\bullet \quad<40$ & 34 & 32.0 & \\
\cline { 1 - 3 }$\bullet \quad 40-80$ & 16 & 0.0 & \\
\hline
\end{tabular}

In our study, 34 patients had neutrophil count of less than $40 \%$ and 16 patients had neutrophils between 40 to $80 \%$. None of the patients had neutrophils more than $80 \%$.

\section{Discussion}

In our study, there were 19 females $(38 \%)$ and 31 males $(62 \%)$. The male to female ratio was $1.6: 1$. In a study by Nagaram PP et al, about 174 confirmed cases of dengue were included with 95 (54.6\%) males and $79(45.4 \%)$ females. Among males, 87 were diagnosed with non-severe dengue cases and 62 among female were non-severe cases. Male to female ratio in our study was $1.2: 1[8]$.

In a study done in Odisha by Mishra et al, there were $75(77.31 \%)$ males and $22(22.68 \%)$ females in [9].

In our study, $26 \%$ of the patients had leucopenia $(<4000), 66 \%$ had leucocyte count in normal range $(4,000-11,000)$ and only $8 \%$ had leucocytosis $(>11,000)$.

In study by Nagaram PP et al leukopenia $(<4000 / \mathrm{mm} 3)$ was observed in $96(55.17 \%)$ cases of study with 3 severe dengue cases and 93 non-severe dengue cases, while 26 cases (14.94\%) had leukocytosis (>11,000/mm3). Normal leukocyte counts were observed in $52(29.89 \%)$ cases with counts between $4000-11000$ cells $/ \mathrm{mm} 3$ with 10 cases of severe dengue and 42 cases of non-severe dengue [8].

A study was done in southern Odisha, to assess the clinical profile of the dengue infection in children less than 14 years of age at the Pediatric Department of Maharaja Krishna Chandra Gajapati Medical College. A total of 97 dengue cases were there. About $58.76 \%$ of the cases had normal leukocyte count, while leucopenia was seen in $25.77 \%$ and leukocytosis in $15.46 \%$ of the cases [9]. In another study from Thailand, 60 children of age group 6 months to 14 years who had acute dengue febrile illness, mild leucopenia was found [10].

In a study done by Christopher et al, a total of 284 patients were enrolled with acute febrile illness. Dengue patients were significantly more likely to have leucopenia (87\%) than any other infection[11].

In another study, out of 99 patients with dengue fever, 44 patients had leucopenia [12]. In another study by Potts, patients with dengue had significantly lower platelet and white blood cell (WBC) [13].

In recent years, it has become evident that neutrophils not only have a fundamental role in the acute phase of inflammation when they actively eliminate pathogens, but also are capable of modifying the overall immune response. Neutrophils can do this by exchanging information with macrophages, dendritic cells, and other cells of the adaptive immune system through either soluble mediators or direct cell-cell contact [12]. 
Neutropenia in dengue infections has also been reported, although less frequently. Neutrophils are important to initiate and maintain an immune response leading to destruction of microorganisms. In general, persons with severe neutropenia as defined as less than $0.5 \times 10^{9} / \mathrm{L}$ are at higher risk of secondary bacterial infections, and clinicians often institute antibiotics prophylactically for patients presenting with severe neutropenia.

However, studies on severe neutropenia in dengue are scarce, and its clinical significance is uncertain. In particular, it is unknown whether dengue patients with severe neutropenia are more prone to secondary bacterial infections and whether prophylactic antibiotics would be justified [14].

In our study, neutrophil count of less than $40 \%$ was found in $34(68 \%)$ indicating neutropenia and 16 (32\%) patients had neutrophils between 40 to $80 \%$. None of the patients had neutrophils more than $80 \%$. Conservative treatment was given to the patients with intravenous fluids and antipyretics. Patients were hemodynamically stable throughout. In patients with neutropenia, prophylactic antibiotics were not used as there were no signs and symptoms of any secondary infection. There was no mortality. This justifies that irrational use of antibiotics is not necessary in all cases of neutropenia in dengue fever. Further studies are needed to determine the rationale of antibiotic usage in dengue.

In another study done in Thailand, about 996 children serologically diagnosed with dengue infection and admitted to the Department of Pediatrics, Chulalongkorn Hospital, Bangkok, Thailand between 1988 and 1995 were retrospectively reviewed. One hundred and thirty-nine cases $(14.0 \%)$ were serologically proved to be primary dengue infection. Of these, 72 were males and 67 were females. In this study maximal percentage of neutrophils were significantly lower in the study group in dengue fever patients [15].

In a study by Thein et al, during hospitalization $1,579(82.2 \%)$ patients had ANC (absolute neutrophil count) $<1.5 \times 10^{9} / \mathrm{L}$. Dengue patients with severe neutropenia did not have an increased risk for nosocomial infection or antibiotic use in this study. These findings suggest that severe neutropenia is not associated with an increased risk of secondary bacterial infections. No guidelines exist to give antibiotics to dengue patients presenting with severe neutropenia. Severe neutropenia was not associated with secondary bacterial infections, prolonged hospital stay, prolonged fever, or fatal outcome. Prophylactic antibiotics are not indicated in patients with severe neutropenia who have otherwise no clinical or laboratory indication for bacterial infection [14].

\section{Conclusion}

According to our study, in early febrile period of dengue fever, majority have normal white blood cell count. Any change in the values of total leucocyte count points towards the progression of the disease towards severity.

It was also found that most of the patients in early phase of dengue fever had neutropenia which was not related to the course of disease and use of prophylactic antibiotics was not done in them. This would prevent the irrational use of antibiotics thereby reducing the incidence of antibiotic resistance.

Contribution: Both the authors equally participated during study process and manuscript preparation.

Funding: Nil, Conflict of interest: Nil.

Ethical approval: The study was approved by the Institutional Ethics Committee

\section{What this study adds to existing knowledge?}

1. In early febrile period of dengue, change in the values of total leucocyte count points towards the progression of the disease towards severity and calls for early intervention.

2. Pediatricians should avoid irrational use of antibiotics in dengue fever with neutropenia and prevent the development of antibiotic resistance in children.

\section{References}

1. Gupta N, Srivastava S, Jain A, Chaturvedi UC. Dengue in India. Indian J Med Res. 2012 Sep; 136 (3): 373-90.

2. WHO. Dengue and dengue haemorrhagic fever. Factsheet No 117, revised May 2008. Geneva, World Health Organization, 2008. Available from http://www.who.int/mediacentre/factsheets/fs117/en 
3. Martina BE, Koraka P, Osterhaus AD. Dengue virus pathogenesis: an integrated view. Clin Microbiol Rev.2009 Oct;22(4):564-81.doi: 10.1128/ CMR.00035-09.

4. CDC.Clinical guidelines for the management of dengueinfection.Revised November 2015.US Department ofhealth and human services.GA. USA. Available from https: //www.cdc. gov/ dengue/ clinicallab /clinical. html. Accessed October 12, 2017.

5. Kurane I. Dengue hemorrhagic fever with specialemphasis on immunopathogenesis. Comp Immunol Microbiol Infect Dis. 2007 Sep; 30 (5-6):329-40. Epub 2007 Jul 23.

6. World Health Organization (WHO). DengueGuidelines for Diagnosis, Treatment, Prevention and Control. Geneva, Switzerland: World Health Organization; 2009. Available from www.who. int/ rpc/guidelines_/9789241547871. Accessed October $12,2017$.

7. Rosales C, Demaurex N, Lowell CA, Uribe QE. Neutrophils: Their Role in Innate and Adaptive Immunity. Journal of Immunology Research. 2016; 2016: 1469780. doi:10.1155/2016/1469780.

8. Nagaram PP, Pidugu P, Munagala VK, Matli VV. Clinical and laboratory profile and outcome of dengue cases among children attending a tertiary care hospital of South India. Int J ContempPediatr. 2017; 4: 1074-80. doi. org/10.18203/2349-3291.ijcp 20171731

9. Mishra S, Ramanathan R, Agarwalla SK. Clinical Profile of Dengue Fever in Children: A Study from Southern Odisha, India. Scientifica (Cairo). 2016; 2016 (4):6391594. doi:10.1155/2016/6391594.
10. KalayanaroojS, Vaughn DW, Nimmannitya S, Green S, Suntayakorn S, Kunentrasai N, Viramitrachai W, Ratanachu-eke S, Kiatpolpoj S, Innis BL, Rothman AL, Nisalak A, Ennis FA. Earlyclinical and laboratory indicators of acuted engueillness. J Infect Dis. 1997 Aug;176(2):313-21.

11. Gregory CJ, Lorenzi OD, Colón L, García AS, Santiago LM, Rivera RC, Bermúdez LJ, Báez FO, Aponte DV, Tomashek KM, Gutierrez J, Alvarado L. Utility of the tourniquet test and the white blood cell count to differentiate dengue among acute febrileillnesses in the emergency room. PLo S Negl Trop Dis. 2011 Dec;5(12): e1400. doi: 10.1371/ journal. pntd.0001400. Epub 2011 Dec 6.

12. Jayanthi HK, Tulasi SK. Correlation study between platelet count, leukocyte count, nonhemorrhagic complications, and duration of hospital stay in dengue fever with thrombocytopenia. Journal of Family Medicine and Primary Care. 2016;5(1):120-123.

13. Potts JA, Rothman AL. Clinical and laboratory features that distinguish dengue from other febrileillnesses in endemic populations. Trop Med Int Health. 2008 Nov; 13(11):1328-40. doi: 10. 1111/j.1365-3156. 2008.02151.x. Epub 2008 Sep 16.

14. Thein TL, Lye DC, Leo YS, Wong JG, Hao Y, Wilder- Smith A. Severeneutropenia in denguepatients: prevalence and significance. Am J Trop Med Hyg. 2014 Jun; 90 (6):984-7. doi: 10.4269 / ajtmh. 14-0004. Epub 2014 Apr 14.

15. Pancharoen C, Mekmullica J, Thisyakorn U. Primary dengue infection: what are the clinical distinctions from secondary infection? Southeast Asian J Trop Med Public Health. 2001;32:476-480.

\section{How to cite this article?}

Khandelwal R, Khandelwal LM. Effect of dengue fever on the total leucocyte count and neutrophil count in children in early febrile period. Int J Pediatr Res. 2017;4(10):617-622.doi:10.17511/ijpr.2017.i10.06. 